\title{
Geological records of past sea-level changes as constraints for future projections
}

\author{
Benjamin P. Horton ${ }^{1,2,3}$, R.E. Kopp ${ }^{3,4}$, A. Dutton ${ }^{5}$ and T.A. Shaw ${ }^{1}$
}

\begin{abstract}
Geological records of sea level have varied in response to a wide range of boundary conditions and climate forcings, and these geological records can serve as a valuable guide to projecting the dynamics of ice-sheet retreat and sealevel rise into the future. However, using geological records in this manner requires a robust understanding of regional variability and rigorous quantification of uncertainty.
\end{abstract}

Sea-level projections depend upon an accurate understanding of the relationship between climate forcing and the spatiotemporal evolution of sea level, as well as its different driving mechanisms. Yet this understanding is limited by the timescale of the instrumental data; most available records contain data only from the 20th and 21 st centuries (Horton et al. 2018). Complementing instrumental data, geological records can provide valuable archives of the sea-level response to past climate variability, including during periods of higher global mean temperatures (Dutton et al. 2015), and can help improve estimates of the relationship between future temperature and sea-level change (Kopp et al. 2016). However, current ties between geological sea-level records and future projections are often vague and tentative; improved interconnections between the two subdisciplines are thus a key to progress (Horton et al. 2018).

Sea-level-rise projections and geological records of past sea-level change share two fundamental challenges. First, regional sealevel changes vary substantially from global mean sea level (GMSL) change (Khan et al. 2015). Understanding regional variability is critical to both interpreting geological records and generating projections for effective coastal risk management. Second, uncertainty, which is pervasive in reconstructions of geological changes and both physical and statistical modeling approaches used to project future changes, requires careful assessment and quantification.

Here, we highlight two case studies - semiempirical models of GMSL rise and estimates of the Antarctic contribution to sea level - to illustrate the way in which geological records can improve future projections, and conversely how the need for improved future projections can guide the development of geological sea-level research questions.

\section{Common Era}

Semi-empirical models use a statistical relationship between past global mean temperature and past GMSL to estimate the response of future sea levels to projected temperatures. Many semi-empirical models link instrumental records of sea level and temperature with multiple mathematical terms (Vermeer and Rahmstorf 2009). For example, the fast-response term is conceptually linked to heat storage in the ocean mixed layer and the slow-response is conceptually linked to the deep ocean and ice sheets. As of the Fifth Assessment Report of the Intergovernmental Panel on Climate Change (IPCC) in 2013, semi-empirical models were almost universally calibrated only to the instrumental record and yielded projections higher than the bottom-up, process-based projections preferred by the IPCC. For example, Schaeffer et al. (2012) provided a median semi-empirical projection of 21st century GMSL rise under a mod erate-emissions pathway (Representative Concentration Pathway (RCP) 4.5) that was $90 \mathrm{~cm}$, compared to the IPCC's median projection of $53 \mathrm{~cm}$ (Church et al. 2013).

The instrumental semi-empirical approach is, however, potentially biased by the short time span of instrumental records, which often post-date the onset of accelerated sealevel rise (Kemp et al. 2011), as well as the presence of substantial decadal and multidecadal sea-level variability that is unrelated to global temperature changes (Horton et al. 2018). One solution to this problem is the use of longer geological records of sea level (Kemp et al. 2011).

Although temperature and sea-level variability over the Common Era (the last 2000 years) are not analogous to future changes, empirical relationships between high-resolution sea-level records can be paired with temperature reconstructions showing periods of both warming and cooling to examine the dynamic response of sea level to climate forcing on multi-decadal to multi-centennial timescales (Kemp et al. 2011). Common Era proxy records using salt-marsh sediment sequences permit the reconstruction of near-continuous time series of decimeterscale sea-level changes over this period. Although Glacial Isostatic Adjustment (GIA) is the dominant cause of regional variability (Fig. 1a), it is approximately linear over the Common Era (Engelhart et al. 2009). Simple linear detrending of long-term local trends can thus circumvent uncertainties induced by parameterizations of GIA models, which are needed to correct earlier reconstructions (Dutton et al. 2015). Furthermore, detrending highlights allows for close examination of decadal to centennial variability in GMSL. However, such detrending masked any small changes in GMSL driven by multimillennial-scale processes other than GIA, and prevents an assessment of whether current GMSL is higher than its peak Common Era level.

Kopp et al. (2016) calibrated a semi-empirical model to a new statistical reconstruction of GMSL and existing global-mean temperature reconstructions. The resulting model indicated that without 20th century global warming, GMSL would have risen by less than $60 \%$ of the observed increase from 1900 to 2000 CE and might even have fallen. This estimate is in reasonable agreement with estimates of the human contribution to 20th century sea-level rise based on process models (Slangen et al. 2016). Subsequently, the projections from the semi-empirical model were substantially lower than those calibrated only to instrumental records and, indeed, reconciled the differences between semi-empirical models and the IPCC's preferred process-based projections. For RCP 4.5, the Kopp et al. (2016) median projection of $21 \mathrm{st}$ century GMSL rise was $51 \mathrm{~cm}$, which is essentially indistinguishable from that of the IPCC. Their $66 \%$ probability interval of 39-69 $\mathrm{cm}$ was in good agreement with the IPCC's "likely" range, 36-71 cm (Fig. 1b).

While the agreement between semiempirical projections and the IPCC's 2013 projections could be viewed as increasing confidence in both, this may be instilling false hope. By construction, the semi-empirical model assumes that the processes driving changes in GMSL over the Common Era are the same as those that will be important in the 21st century, yet the rapid ongoing changes in polar ice sheets suggests that they may not be. Perhaps the agreement is indicating instead that the process models that informed the 2013 IPCC assessment are implicitly exhibiting the same historical 

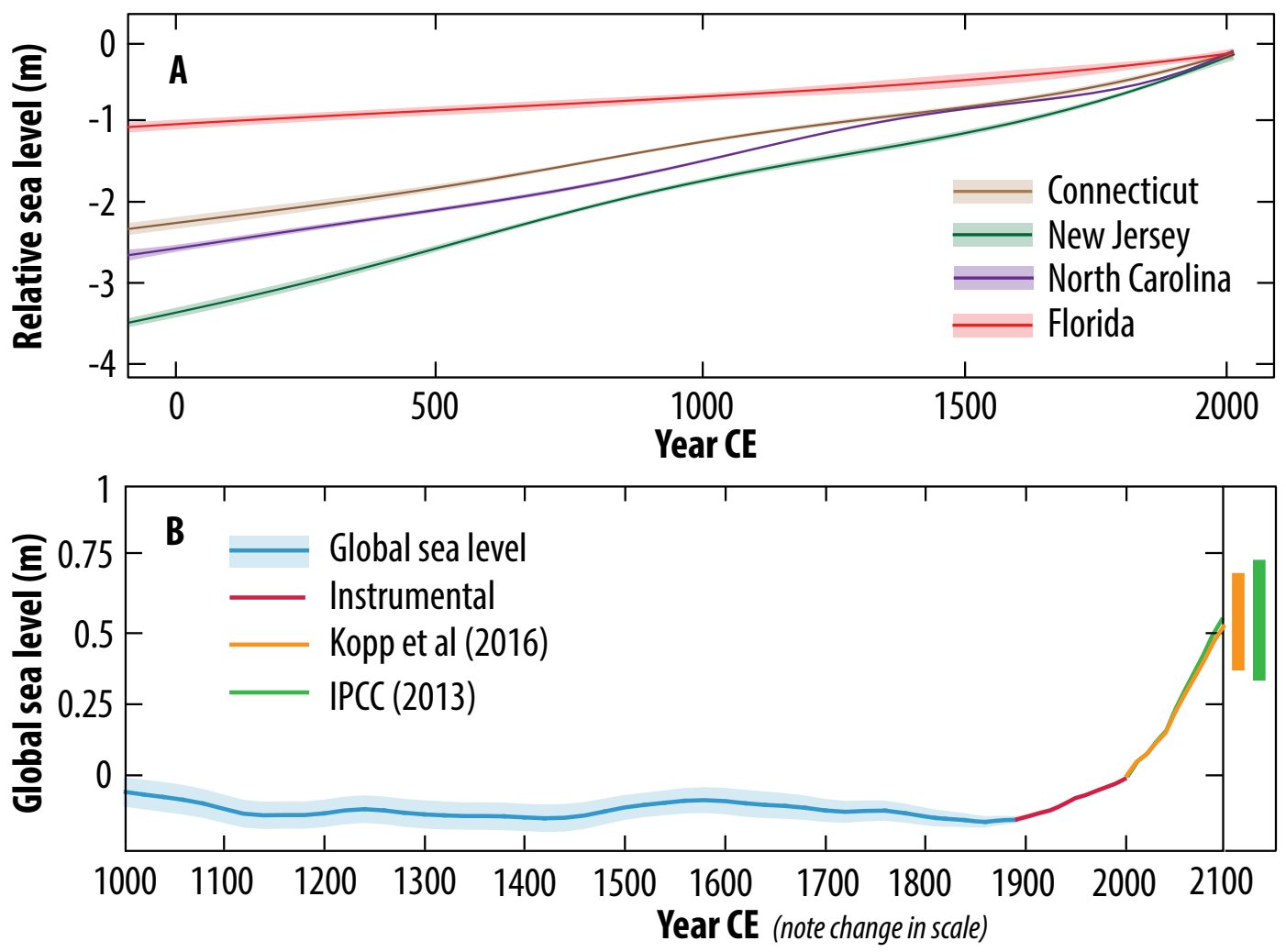

Figure 1: (A) Common Era relative sea-level change on the U.S. Atlantic coast (Kemp et al. 2015 and references therein). (B) Global sea level for the past millennia and $21 \mathrm{st}$ century semi-empirical model projections for RCP 4.5 (Kopp et al. 2016). Orange and green bars show the 5th-95th percentile of RCP 4.5 for semi-empirical (Kopp et al. 2016) and process-based (Church et al. 2013) models, respectively.

biases that are explicit in the semi-empirical model.

\section{Last Interglacial (LIG) and Mid- Pliocene Warm Period (MPWP)}

Geological records from the LIG $(\sim 128,000$ 116,000 years ago) and MPWP ( 3.2-3.0 million years ago) provide alternative sources of constraints on GMSL change in periods with higher temperatures (Dutton et al. 2015). In particular, these past warm climate intervals can shed light on the dynamics of potential rapid destabilization of sectors of the Antarctic ice sheet that remain poorly constrained in current ice-sheet models. Indeed, after the uncertainty in future greenhouse gas emissions, the possible instability of marine-based sectors of the Antarctic ice sheet is the largest driver of uncertainty in future sea-level-rise projections (Kopp et al. 2017). While no particular past warm interval is a perfect analogue of the present climate forcing, many offer insight into thresholds, time lags between forcing and response, and potential rates of ice-sheet retreat (Dutton et al. 2015).

For the LIG, current estimates of peak GMSL rise indicate that it is extremely likely that it exceeded 6 m (Kopp et al. 2009). Yet while it is generally accepted that sea level during both the LIG and the Pliocene were higher than present, precisely quantifying the magnitude of GMSL rise has proven challenging (Horton et al. 2018). Chronological and vertical uncertainties, and potentially significant contributions from GIA and mantle dynamic topography (Austermann et al. 2017), hinder accurate interpretations of geological data, especially for the Pliocene. Moreover, rates of sea-level change for the LIG and MPWP range from highly uncertain to completely unconstrained depending on the time period (Dutton et al. 2015).

DeConto and Pollard (2016) used both LIG and MPWP constraints to calibrate their continental-scale model of the Antarctic ice sheet. In their model, LIG behavior is correlated with sea-level contributions under low-emissions scenarios in the short term (i.e. until $2100 \mathrm{CE}$ ), while MPWP behavior is more indicative of behavior under highemissions scenarios, especially in the long term (i.e. centuries into the future). However, due in part to the large uncertainties that still exist in LIG and MPWP sea-level reconstructions, there is still ample room for re-interpretation of the geological records, and, consequently, the constraints they provide to future projections of sea-level rise.

These types of exercises, where geological sea-level records are employed to constrain the physical parameterization of the icesheet models, help to clarify some important targets for the geological sea-level community. To improve future projections, a key target is to better quantify the magnitude of peak GMSL, which in turn heavily relies upon improving the solid Earth models employed in modeling GIA and dynamic topography. A second key target is establishing both the timing of meltwater input from both Greenland and Antarctica, and constraining the climatic contexts associated with these events.

Ultimately, the fundamental observation from the LIG and MPWP that can be carried over to the present day is that ice sheets (and sea level) have the potential to respond rapidly and non-linearly to warming temperatures. This knowledge should empower us to act now and prepare for a waterier future as global mean temperatures and sea levels continue to rise.

\section{AFFILIATIONS}

'Asian School of the Environment, Nanyang Technological University, Singapore

${ }^{2}$ Earth Observatory of Singapore, Nanyang Technological University, Singapore

Institute of Earth, Ocean, and Atmospheric Sciences, Rutgers University, New Brunswick, NJ, USA

${ }^{4}$ Department of Earth and Planetary Sciences, Rutgers University, Piscataway, NJ, USA

${ }^{5}$ Department of Geological Sciences, University of Florida, Gainesville, USA

\section{CONTACT}

Benjamin P. Horton: bphorton@ntu.edu.sg REFERENCES

Austermann J et al. (2017) Sci Adv 3: e1700457

Church JA et al. (2013) In: Stocker TF et al. (Eds) Climate Change 2013: The Physical Science Basis. Cambridge University Press, 1137-1216

DeConto RM, Pollard D (2016) Nature 531: 591-597

Dutton A et al. (2015) Science 349: aaa4019

Engelhart SE et al. (2009) Geology 37: 1115-1118 Horton BP et al. (2018) Annu Rev Environ Resour 43: 481-521

Kemp AC et al. (2011) PNAS 108: 11017-11022 Kemp AC et al. (2015) Earth Planet Sci Lett 428: 217-229 Khan NS et al. (2015) Curr Clim Change Rep 1: 247-262 Kopp RE et al. (2009) Nature 462: 863-867

Kopp RE et al. (2016) PNAS 113: E1434-E1441 Kopp RE et al. (2017) ArXiv: 1704.05597 Schaeffer M et al. (2012) Nat Clim Change 2: 867-870 Slangen ABA et al. (2016) Nat Clim Change 6: 701-705 Vermeer M, RahmstorfS (2009) PNAS 106: 21527-21532 